\title{
OPERATOR INEQUALITIES, GEODESICS AND INTERPOLATION
}

\author{
GUSTAVO CORACH \\ Instituto Argentino de Matemática \\ Viamonte 1636, 1055 Buenos Aires, Argentina \\ Departamento de Matemática, Facultad de Ciencias Exactas y Naturales \\ Ciudad Universitaria, 1428 Buenos Aires, Argentina
}

Introduction. The topological structure of certain subsets of operator algebras has been studied since the beginning of spectral theory. However, the viewpoint of differential geometry has only recently been adopted. Several papers, most of them included in the references, have appeared in the last years which describe geometrical invariants of certain sets of operators: projections, selfadjoint invertible operators, positive invertible operators, relatively regular operators, and so on. More generally, one can study the geometry of sets of spectral measures, $C^{*}$-algebra and group representations, completely bounded maps between $C^{*}$-algebras, and so on. It turns out that some deep results, e.g. Haagerup's theorem on the similarity orbit of cyclic representations [32], find a natural setting in this geometrical viewpoint (see [3]).

This paper surveys the results discussed in those works. We omit most proofs but we present some simplifications of the original presentations. There is a rather complete panorama of the work of E. Andruchow, A. Maestripieri, H. Porta, L. Recht, D. Stojanoff and the author, but we also discuss contributions by C. J. Atkin, B. Gramsch, K. Lorentz, M. Martin, S. Semmes and D. R. Wilkins. Warning: even when we study differential geometry of certain subsets of noncommutative $C^{*}$-algebras, this paper contains no results on Connes' non-commutative differential geometry.

1991 Mathematics Subject Classification: 46L99, 46B70, 58B20.

The paper is in final form and no version of it will be published elsewhere.

The author gratefully acknowledges the support of the Fundación Antorchas and the International Mathematical Union. 
The contents of the paper are as follows: the first section describes the geometry of the space $G^{\text {s }}$ of selfadjoint invertible elements of a $C^{*}$-algebra; the second section contains a more precise description of the set $G^{+}$of positive invertible elements of $G^{\mathrm{s}}$, which is the connected component of the identity in $G^{\text {s }}$; an interesting fact is that some well known operator inequalities (one of them is known as Segal's inequality) are equivalent to geometric properties of $G^{+}$; the third section discusses the curvature tensor of $G^{\text {s }}$; in Section 4, we relate the geometry of $G^{+}$to the operator means theory, due to Anderson, Trapp, Pusz, Woronowicz, Kubo and Ando, among others; in Section 5 a brief description of the geometry of the space of projections of a $C^{*}$-algebra is given; in Section 6 we show that the geometric study of $G^{+}$is closely related to the quadratic and complex interpolation methods; finally, Section 7 contains a brief survey on several spaces of operators which also have a rich geometrical structure.

Acknowledgements. We thank Prof. G. K. Pedersen for his permission to include his proof of Proposition 1.5. We are also grateful to Prof. J. Zemánek who has communicated to us several bibliographical comments.

Notations. In this paper $\mathcal{A}$ denotes a $C^{*}$-algebra with unit, $G$ is the group of invertible elements of $\mathcal{A}, \mathcal{U}$ is the group of unitary elements and $G^{+}$is the set of positive elements of $G$. For a differentiable map $f: X \rightarrow Y$ and a point $x$ of $X$, $(T f)_{x}$ is the tangent map of $f$ at $x$ which maps the tangent space $(T X)_{x}$ into the tangent space $(T Y)_{f(x)}$. Because every manifold considered here is a submanifold of some Banach space, one can realize a tangent vector as the velocity vector of a differentiable curve in the manifold. In any case, tangent spaces are identified with subspaces of the corresponding Banach space. For all geometric notions we follow the book of Kobayashi and Nomizu [35].

1. The geometry of the set of selfadjoint invertible elements. Let $G^{\mathrm{s}}=G^{\mathrm{s}}(\mathcal{A})$ be the set of all selfadjoint invertible elements of a unital $C^{*}$-algebra $\mathcal{A}$ and consider the action of $G$ on $G^{\mathrm{s}}$ defined by

$$
L_{g} a=g \cdot a=g a g^{*} \quad\left(g \in G, a \in G^{\mathrm{s}}\right) .
$$

For a fixed $a \in G^{\mathrm{s}}$, the orbit

$$
G^{\mathrm{s}, a}=\left\{g a g^{*}: g \in G\right\}
$$

is open and closed in $G^{\mathrm{s}}$ and the map $g \mapsto g a g^{*}$ defines a principal fiber bundle over $G^{\mathrm{s}, a}$ with structure group $U_{a}=\left\{u \in G: u^{-1}=a^{-1} u^{*} a\right\}$. A smooth local section near $a$ can be defined by means of $b \mapsto\left(b a^{-1}\right)^{1 / 2}$, where $x^{1 / 2}$ is the usual square root defined by the power series

$$
\sum_{n=1}^{\infty}\left(\begin{array}{c}
1 / 2 \\
n
\end{array}\right) x^{n} .
$$


$G^{\text {s }}$ has a natural structure as a real-analytic submanifold of $\mathcal{A}$. Since $G^{\mathrm{s}}$ is an open subset of $\mathcal{A}^{\mathrm{s}}=\left\{x \in \mathcal{A}: x^{*}=x\right\},\left(T G^{\mathrm{s}}\right)_{a}$ can be identified with $\mathcal{A}^{\mathrm{s}}$ itself, for every $a \in G^{\mathrm{s}}$.

In the sequel we shall consider the submanifold $P$ of $G^{\mathrm{s}}$ defined by

$$
P=\left\{\varrho \in \mathcal{A}: \varrho^{*}=\varrho=\varrho^{-1}\right\} .
$$

Observe that, for each $\varrho \in P$,

$$
(T P)_{\varrho}=\left\{X \in \mathcal{A}^{\mathrm{s}}: \varrho X=-X \varrho\right\} .
$$

If we consider the restriction to $P$ of the tangent bundle $T G^{\mathrm{s}}, T P$ is a subbundle of $\left.T G^{\mathrm{s}}\right|_{P}$ with a natural complement $N$ given by $N_{\varrho}=\left\{X \in \mathcal{A}^{\mathrm{s}}: \varrho X=X \varrho\right\}$. We shall pursue this issue below.

Given a differentiable curve $\gamma:[u, v] \rightarrow G^{\mathrm{s}, a}$, the fibration properties of $\pi$ : $G \rightarrow G^{\mathrm{s}, a}$ show that there exist curves $\Gamma:[u, v] \rightarrow G$ such that $\pi(\Gamma(t))=\gamma(t)$ $(t \in[u, v])$ (see [49] and [35]). A particular choice of such a $\Gamma$ can be made by solving the differential problem

$$
\left\{\begin{array}{l}
\dot{\Gamma}=\frac{1}{2} \dot{\gamma} \gamma^{-1} \Gamma, \\
\Gamma(u)=1
\end{array}\right.
$$

In fact, differentiating $\Gamma^{-1} \gamma \Gamma^{*-1}$ gives

$$
\begin{aligned}
\left(\Gamma^{-1} \gamma \Gamma^{*-1}\right)^{\cdot} & =-\Gamma^{-1} \dot{\Gamma} \Gamma^{-1} \gamma \Gamma^{*-1}+\Gamma^{-1} \dot{\gamma} \Gamma^{*-1}-\Gamma^{-1} \gamma \Gamma^{*-1} \dot{\Gamma}^{*} \Gamma^{*-1} \\
& =-\Gamma^{-1}\left(\frac{1}{2} \dot{\gamma} \gamma^{-1} \gamma-\dot{\gamma}+\frac{1}{2} \gamma \gamma^{-1} \dot{\gamma}\right) \Gamma^{*-1}=0
\end{aligned}
$$

so that $\Gamma^{-1} \gamma \Gamma^{*-1} \equiv\left(\Gamma^{-1} \gamma \Gamma^{*-1}\right)(u)=a$.

We shall see that $\Gamma$ has a very precise geometrical meaning.

The differential equation appears naturally. In fact, given a partition $\Pi$ of the interval $[u, t]$ one gets, using the local section mentioned above, an invertible element $g=g_{\Pi}$ such that $g \cdot \gamma(u)=\gamma(t)$. It turns out that the limit $\Gamma(t)=$ $\lim _{\|\Pi\| \rightarrow 0} g_{\Pi}$ exists, $\Gamma(t)$ is invertible and the curve $\Gamma$ is differentiable and satisfies $\dot{\Gamma}=\frac{1}{2} \dot{\gamma} \gamma^{-1} \Gamma$ (see [16] for details).

There is a natural connection on the principal bundle $\pi_{a}: G \rightarrow G^{\mathrm{s}, a}$. In fact, the Lie algebra of the group $U_{a}=\left\{u \in G: u^{-1}=a^{-1} u^{*} a\right\}$ is $\mathcal{U}_{a}=\{X \in \mathcal{A}$ : $\left.a^{-1} X^{*} a=-X\right\}$, which decomposes $\mathcal{A}=T G_{1}$ as

$$
\mathcal{A}=\mathcal{U}_{a} \oplus S_{a},
$$

where $S_{a}=\left\{X \in \mathcal{A}: a^{-1} X^{*} a=X\right\}$. Then, if we identify $\mathcal{A}$ with the tangent space of $G$ at $1,(T G)_{1}$, the map $g \mapsto H_{g}=\left\{g X: X \in S_{a}\right\}$ defines a $C^{\infty}$ distribution of horizontal spaces for a connection on $\pi: G \rightarrow G^{\mathrm{s}, a}$ : for this (see [35]) it suffices to check that $H_{g} u=H_{g u}$ for all $u \in U_{a}$ and $g \in G$, which is equivalent to proving that $u S_{a} u^{-1} \subset S_{a}$ for all $u \in U_{a}$ and this inclusion is easily verified.

For every $C^{1}$ curve $\gamma:[0,1] \rightarrow G^{\mathrm{s}, a}$ with origin $a$ there is a unique curve $\Gamma$ in $G$ such that $\Gamma(t) a \Gamma(t)^{*}=\gamma(t), \Gamma(0)=1$ and $\dot{\Gamma}(t) \in H_{\Gamma(t)}$ (this is called the 
horizontal lift of $\gamma$ with origin 1). It turns out that $\Gamma$ is precisely the solution of the differential equation discussed above (see [16], 2.3). is

If $X$ is a tangent field along a curve $\gamma$ in $G^{\mathrm{s}, a}$, the covariant derivative of $X$

$$
\frac{D X}{d t}=\dot{X}-\frac{1}{2}\left(X \gamma^{-1} \dot{\gamma}+\dot{\gamma} \gamma^{-1} X\right)
$$

This definition comes from the computation of the derivative of the tangent map of $L_{\Gamma^{-1}(t)}$ at the point $\gamma(t)$ applied to $X(t)$ :

$$
\left.\frac{D X}{d t}\right|_{t=t_{0}}=\left.\frac{d}{d t}\right|_{t=t_{0}}\left(T L_{\Gamma^{-1}(t)}\right)_{\gamma(t)} X(t),
$$

where $\Gamma$ is the solution of $\dot{\Gamma}=-\frac{1}{2} \gamma^{-1} \dot{\gamma} \Gamma$ with $\Gamma\left(t_{0}\right)=1$.

Recall that a field $X$ is parallel if $D X / d t=0$, and a curve $\gamma$ is a geodesic if $\dot{\gamma}$ is parallel, i.e. if $\gamma$ satisfies

$$
\ddot{\gamma}=\dot{\gamma} \gamma^{-1} \dot{\gamma}
$$

The connection on the tangent bundle is $G$-invariant, in the sense that $\gamma$ is a geodesic if and only if $g \gamma g^{*}$ is a geodesic for all $g \in G$. The unique geodesic $\gamma$ such that $\gamma(0)=a$ and $\dot{\gamma}(0)=X \in\left(T G^{\mathrm{s}}\right)_{a}$ is easily seen to be

$$
\gamma(t)=e^{(t / 2) X a^{-1}} \cdot a=e^{(t / 2) X a^{-1}} a e^{(t / 2) a^{-1} X}=e^{t X a^{-1}} a=a e^{t a^{-1} X}
$$

(recall that $g e^{z} g^{-1}=e^{g z g^{-1}}$ ).

We do not know under what conditions $G^{\mathrm{s}, a}$ is geodesically complete. We shall show, however, a large family of geodesically complete submanifolds of $G^{\mathrm{s}}$.

The curvature tensor of $T G^{\mathrm{s}}$ is given by

$$
R(X, Y) Z=\frac{1}{4}\left(Z\left[a^{-1} X, a^{-1} Y\right]-\left[X a^{-1}, Y a^{-1}\right] Z\right)
$$

where $X, Y, Z \in\left(T G^{\mathrm{s}}\right)_{a}$ and $[V, W]=V W-W V$. We shall determine later some spectral properties of $R$.

Given an invertible operator $T$ on a Hilbert space $\mathcal{H}$ there exist the so-called polar decompositions of $T$ :

$$
T=V P=P^{\prime} V^{\prime}
$$

where $V, V^{\prime}$ are unitary and $P, P^{\prime}$ are positive operators; moreover, $P$ is the (unique) positive square root of $T^{*} T, P^{\prime}$ is the square root of $T T^{*}$ and $V=V^{\prime}=$ $T\left(T^{*} T\right)^{-1 / 2}=\left(T T^{*}\right)^{-1 / 2} T$. Observe that because $V$ and $P$ depend analytically on $T$, they belong to the closed $C^{*}$-subalgebra generated by $I$ and $T$. It follows that, given a $C^{*}$-algebra $\mathcal{A}$ we can consider the analytic map $\pi: G \rightarrow \mathcal{U}$ defined by $\pi(g)=u$ if $g=\lambda u, \lambda \in G^{+}, u \in \mathcal{U}$.

Observe that $\pi(a)^{*}=\pi(a)=\pi(a)^{-1}$ if $a \in G^{\mathrm{s}}$ : in fact, if $a=\lambda \varrho, \lambda \in G^{+}$, $\varrho \in \mathcal{U}$ then $a=a^{*}=\varrho^{-1} \lambda$, but by the unicity argument discussed above, $\varrho^{-1}=\varrho$ and, in consequence, $\lambda \varrho=\varrho \lambda$. In particular, $\pi(a) \in P$ if $a \in G^{\mathrm{s}}$. Thus, we get the map

$$
\pi: G^{\mathrm{s}} \rightarrow P, \quad \pi(\lambda \varrho)=\varrho
$$


We shall study more carefully the fibers $G_{\varrho}^{\mathrm{s}}=\pi^{-1}(\varrho)$.

Observe that, if $\varrho^{\prime}=\{X \in \mathcal{A}: X \varrho=\varrho X\}$, then

$$
G_{\varrho}^{\mathrm{s}}=\{\lambda \varrho: \lambda \varrho=\varrho \lambda, \lambda>0\}=G^{+}\left(\varrho^{\prime}\right) \varrho .
$$

Thus, in order to know the geometry of the fibers $G_{\varrho}^{\mathrm{s}}$ it suffices to know the geometry of $G^{+}(\mathcal{B})$ for any unital $C^{*}$-algebra $\mathcal{B}$.

Observe that the group $G\left(\varrho^{\prime}\right)=\{g \in G: g \varrho=\varrho g\}$ acts transitively on $G_{\varrho}^{\mathrm{s}}$ : in fact, if $\lambda \varrho=\varrho \lambda$ and $\mu \varrho=\varrho \mu$ with $\lambda, \mu \in G^{+}$then $g=\mu^{1 / 2} \lambda^{-1 / 2}$ belongs to $G\left(\varrho^{\prime}\right)$ and $g \lambda \varrho g^{*}=\mu \varrho$.

A Finsler structure can be defined on $G^{\mathrm{s}}$ as follows: if $a \in G^{\mathrm{s}}$ and $X \in\left(T G^{\mathrm{s}}\right)_{a}$, the decomposition $a=\lambda \varrho$ provides a norm \|\|$_{a}$ in $\left(T G^{\mathrm{s}}\right)_{a}$ by means of

$$
\|X\|_{a}=\left\|\lambda^{-1 / 2} X \lambda^{-1 / 2}\right\| \text {. }
$$

In particular, $\|X\|_{\varrho}=\|X\|$ if $\varrho \in P$.

The meaning of this definition is the following. If $\mathcal{A}$ is represented in a Hilbert space $\mathcal{H},\|X\|_{a}$ is the norm of the symmetric sesquilinear form $B_{X}: \mathcal{H}_{a} \times \mathcal{H}_{a} \rightarrow \mathbb{C}$, $B_{X}(\xi, \eta)=\langle X \xi, \eta\rangle$ where $\mathcal{H}_{a}$ is the space $\mathcal{H}$ with the scalar product $\langle\xi, \eta\rangle_{a}=$ $\langle\lambda \xi, \eta\rangle$. Observe that if $g$ commutes with $\varrho$ then the polar decomposition of $g a g^{*}$, where $a=\lambda \varrho$, is $\left(g \lambda g^{*}\right) \varrho$. Then $g: \mathcal{H}_{g a g^{*}} \rightarrow \mathcal{H}_{a}$ is an isometry. This implies that $G\left(\varrho^{\prime}\right)$ acts isometrically on $G_{\varrho}^{\mathrm{s}}$, i.e. for every $X \in\left(T G^{\mathrm{s}}\right)_{a}$ and $g \in G\left(\varrho^{\prime}\right)$ it follows that

$$
\left\|g X g^{*}\right\|_{g a g^{*}}=\|X\|_{a} .
$$

In fact, $\left\|g X g^{*}\right\|_{g a g^{*}}$ is the norm of $B_{g X g^{*}}: \mathcal{H}_{g a g^{*}} \times \mathcal{H}_{\text {gag }} \rightarrow \mathbb{C}$ and, because $g: \mathcal{H}_{\text {gag* }} \rightarrow \mathcal{H}_{a}$ is an isometry, $\left\|B_{g X g^{*}}\right\|=\left\|B_{X}\right\|$, which proves the assertion.

Observe that if $a \in G^{+}$then $\varrho=1$ so that $\varrho^{\prime}=\mathcal{A}$. Thus, if $a \in G^{+}$then (1.1) holds for every $X \in \mathcal{A}^{\mathrm{s}}$ and $g \in G$. In particular, $G$ acts isometrically and transitively on $G^{+}$.

The next result contains a complete description of the geometry of the fibers $G_{\varrho}^{\mathrm{s}}$.

Theorem 1.2. For every $\varrho \in P$ let $N_{\varrho}=\left\{X \in\left(T G^{\mathrm{s}}\right)_{\varrho}: X \varrho=\varrho X\right\}$. Then

(i) $N=\left\{(\varrho, X): X \in N_{\varrho}\right\}$ is a subbundle of $\left.T G^{\mathrm{s}}\right|_{P}$ such that $\left.T G^{\mathrm{s}}\right|_{P}=$ $T P \oplus N$.

(ii) The restriction to $N$ of the exponential map

$$
\exp _{a} X=e^{(1 / 2) a X} a e^{(1 / 2) X a} \quad\left(a \in G^{\mathrm{s}}, X \in\left(T G^{\mathrm{s}}\right)_{a}\right)
$$

is a diffeomorphism $\theta: N \rightarrow G^{\mathrm{s}}$ such that $\theta\left(N_{\varrho}\right)=G_{\varrho}^{\mathrm{s}}=\pi^{-1}(\varrho)$ and $\theta^{-1}(\lambda \varrho)=$ $(\varrho, \varrho \log \lambda)$.

(iii) Any two points in $G_{\varrho}^{\mathrm{s}}$ are joined by a unique geodesic entirely contained in $G_{\varrho}^{\mathrm{s}}$; this geodesic is the shortest curve in $G^{\mathrm{s}}$ joining them.

The proof of this theorem and the main part of the results on $G^{\mathrm{s}}$ described in this section are contained in the paper [16]. We shall indicate, however, a short proof of (iii), the non-standard part of the theorem, in the next section. 
It is interesting to notice that the fact that the geodesics in $G_{\varrho}^{\mathrm{s}}$ are short curves strongly depends on the following contractive property of $\pi$ : the tangent map of $\pi: G^{\mathrm{s}} \rightarrow P$ decreases norms, i.e.

$$
\left\|(T \pi)_{a} X\right\| \leq\|X\|_{a}
$$

for all $a \in G^{\mathrm{s}}$ and $X \in\left(T G^{\mathrm{s}}\right)_{a}$. The inequality (1.3) is equivalent to

$$
\left\|S T S^{-1}+S^{-1} T S\right\| \geq 2\|T\|,
$$

for any selfadjoint operator $S$ and any bounded operator $T$. The original proof of this inequality, published in [17], reduces to the finite-dimensional case, where a Schur product result due to Davis [23] and Walter [51], together with an elementary equality for determinants from Pólya and Szegö [43], do the job.

More recently G. K. Pedersen proved the following result, which generalizes the inequality and has a shorter proof.

Proposition 1.5. For every bounded operator $S, T$ on a Hilbert space $\mathcal{H}$ with $S$ invertible,

$$
\left\|S T S^{-1}+\left(S^{*}\right)^{-1} T S^{*}\right\| \geq 2\|T\| .
$$

Pr o of. First, suppose that $T^{*}=T$. Recall that $\operatorname{Re}(A)=\frac{1}{2}\left(A+A^{*}\right)$. Then, if $r(T)=\sup \{|\lambda|: \lambda \in \sigma(T)\}$, we have

$$
\begin{aligned}
\|T\| & =r(T)=r\left(S T S^{-1}\right) \leq \sup _{|\xi| \leq 1}\left|\operatorname{Re}\left\langle S T S^{-1} \xi, \xi\right\rangle\right|=\sup _{|\xi| \leq 1}\left|\left\langle\operatorname{Re}\left(S T S^{-1}\right) \xi, \xi\right\rangle\right| \\
& =\left\|\operatorname{Re}\left(S T S^{-1}\right)\right\|=\frac{1}{2}\left\|S T S^{-1}+S^{*-1} T S^{*}\right\| .
\end{aligned}
$$

In the general case, put

$$
\widetilde{S}=\left(\begin{array}{cc}
S & 0 \\
0 & S
\end{array}\right) \quad \text { and } \quad \widetilde{T}=\left(\begin{array}{cc}
0 & T^{*} \\
T & 0
\end{array}\right)
$$

on $\mathcal{H} \oplus \mathcal{H}$ and $A=S T S^{-1}+S^{*-1} T S^{*}$ on $\mathcal{H}$. Then

$$
\widetilde{S} \widetilde{T} \widetilde{S}^{-1}+\widetilde{S}^{*-1} \widetilde{T} \widetilde{S}^{*}=\left(\begin{array}{cc}
0 & A^{*} \\
A & 0
\end{array}\right)
$$

and the result follows from the first case.

Added in proof. In [26], the authors prove that inequality (1.4) is equivalent to the following:

$$
\left\|S T R^{-1}+S^{-1} T R\right\| \geq 2\|T\|
$$

for every selfadjoint invertible $S$ and $R$. Indeed, by combining this fact with the proof of 1.5 , it can easily be seen that the inequalities are also equivalent to

$$
\left\|S T R+S^{*-1} T R^{*-1}\right\| \geq 2\|T\|
$$

for any invertible operators $S$ and $R$.

2. The geometry of the positive invertible elements. We now consider the set $G^{+}$. There are, at least, two natural ways of thinking of $G^{+}$with respect 
to $\left.G^{\mathrm{s}}: 1\right)$ as the connected component of the identity; 2) as the fiber $\pi^{-1}(1)$. The first way allows us to study the fiber bundles

$$
G \rightarrow G^{+}, \quad g \mapsto g a g^{*}
$$

(for any $a \in G^{+}$). Thus we can lift any differentiable curve $\gamma$ in $G^{+}$by means of the differential equation

$$
\dot{\Gamma}=\frac{1}{2} \dot{\gamma} \gamma^{-1} \Gamma
$$

The second way gives us some geometric information on $G^{+}$. Observe that $G^{+}$is an open convex subset of $\mathcal{A}^{\mathrm{s}}$, but the geometry we have introduced above is quite different from that induced by $\mathcal{A}^{\mathrm{s}}$. For this, we first determine the geodesics of $G^{+}$. Given $a, b$ in $G^{+}$the (unique) geodesic $\gamma$ such that $\gamma(0)=a$ and $\gamma(1)=b$ is $\gamma(t)=a^{1 / 2}\left(a^{-1 / 2} b a^{1 / 2}\right)^{t} a^{1 / 2}$ : indeed, $\gamma$ satisfies $\ddot{\gamma}=\dot{\gamma} \gamma^{-1} \dot{\gamma}$ and it has the right values at 0 and at 1 . If we want to present $\gamma$ in the form $e^{t X a^{-1}} a$, it suffices to take $X=a^{1 / 2} \log \left(a^{-1 / 2} b a^{1 / 2}\right) a^{-1 / 2}$.

The geodesic distance between $a$ and $b$ is, by definition, the number

$$
d(a, b)=\inf \left\{\text { length } \gamma: \gamma \text { is a } C^{1} \text { curve that } \gamma(0)=a \text { and } \gamma(1)=b\right\} ;
$$

by Theorem 1.1, $d(a, b)$ is exactly the length of

$$
\gamma(t)=a^{1 / 2}\left(a^{-1 / 2} b a^{-1 / 2}\right)^{t} a^{1 / 2} .
$$

Now, length $\gamma=\int_{0}^{1}\|\dot{\gamma}(t)\|_{\gamma(t)} d t=\left\|\log \left(a^{-1 / 2} b a^{-1 / 2}\right)\right\|$; the last equality is proved in the next lines (see [19], Proposition 2 for a discussion of the last formula).

Now we can determine the geodesics in the fibers $G_{\varrho}^{\mathrm{s}} \subset G^{\mathrm{s}}$. Given $a, b$ in $\pi^{-1}(\varrho)$, we write their polar decompositions $a=\lambda \varrho, b=\mu \varrho$ and observe that $\lambda=|a|=\left(a^{2}\right)^{1 / 2}$ and $\mu=|b|=\left(b^{2}\right)^{1 / 2}$ commute with $\varrho$. Then $\gamma(t)=$ $\lambda^{1 / 2}\left(\lambda^{-1 / 2} \mu \lambda^{-1 / 2}\right)^{t} \lambda^{1 / 2} \varrho$ is a geodesic entirely contained in $G_{\varrho}^{\mathrm{s}}$ which satisfies $\gamma(0)=a, \gamma(1)=b$ so that $d(a, b)=$ length $\gamma=\int_{0}^{1}\|\dot{\gamma}(t)\|_{\gamma(t)} d t$. In order to calculate the integral, let us abbreviate $\nu=\lambda^{-1 / 2} \mu \lambda^{-1 / 2}$. Then $\gamma(t)=\lambda^{1 / 2} \nu^{t} \lambda^{1 / 2} \varrho$, $\dot{\gamma}(t)=\lambda^{1 / 2}(\log \nu) \nu^{t} \lambda^{1 / 2} \varrho$ and

$$
\begin{aligned}
\|\dot{\gamma}(t)\|_{\gamma(t)} & =\left\|\lambda^{1 / 2}(\log \nu) \nu^{t} \lambda^{1 / 2}\right\|_{\lambda^{1 / 2} \nu^{t} \lambda^{1 / 2}}=\left\|(\log \nu) \nu^{t}\right\|_{\nu^{t}} \\
& =\left\|\nu^{t / 2}(\log \nu) \nu^{t / 2}\right\|_{\nu^{t}}=\|\log \nu\| .
\end{aligned}
$$

(We have repeatedly used the fact that $G$ acts isometrically on $G^{+}$.)

This shows that

$$
d_{G^{\mathrm{s}}}(a, b)=d_{G^{+}}(|a|,|b|)=\left\|\log \left(\lambda^{-1 / 2} \mu \lambda^{-1 / 2}\right)\right\| .
$$

Let us discuss more carefully some properties of $G^{+}$.

Let $a \in G^{+}$and consider the exponential map $\exp _{a}:\left(T G^{+}\right)_{a} \rightarrow G^{+}$defined by

$$
\exp _{a}(X)=e^{X a^{-1}} \cdot a=e^{(1 / 2) X a^{-1}} a e^{(1 / 2) a^{-1} X}=a^{1 / 2} e^{a^{-1 / 2} X a^{-1 / 2}} a^{1 / 2} .
$$

It is easy to see that $\exp _{a}$ is a diffeomorphism whose inverse $\log _{a}$ is given by

$$
\log _{a}: G^{+} \rightarrow\left(T G^{+}\right)_{a}, \quad \log _{a} b=a^{1 / 2} \log \left(a^{-1 / 2} b a^{-1 / 2}\right) a^{1 / 2} .
$$


These maps lead us to compare the distances in $\left(T G^{+}\right)_{a}$ with the distances in $G^{+}$, i.e. $\|X-Y\|_{a}$ with $d\left(\exp _{a} X, \exp _{a} Y\right)$ for any $X, Y \in\left(T G^{+}\right)_{a}$. It turns out that

$$
d\left(\exp _{a} X, \exp _{a} Y\right) \geq\|X-Y\|_{a} .
$$

(This is a property that $G^{+}$shares with Riemannian manifolds of non-positive curvature.) Observe that (2.2) shows that $\exp _{a}$ increases lengths of curves. An interesting fact proved in [18] is that the inequality above is equivalent to the operator inequality

$$
\left\|e^{X+Y}\right\| \leq\left\|e^{X / 2} e^{Y} e^{X / 2}\right\|
$$

(valid for all selfadjoint operators $X, Y$ on a Hilbert space), which has been proven by I. Segal in [46] using the fact that, for positive operators, taking the positive square root is a monotone operation: $0 \leq A \leq B$ implies $A^{1 / 2} \leq B^{1 / 2}$. Segal's proof holds for not necessarily bounded operators but in our context bounded operators suffice.

There is a beautiful theory which studies the so-called Pick functions: these are real functions $f:[a, b] \rightarrow \mathbb{R}$ which admit an analytic continuation $\widetilde{f}$ to the upper semiplane $\mathbb{C}_{+}$such that $\widetilde{f}\left(\mathbb{C}_{+}\right) \subset \mathbb{C}_{+}$. K. Löwner [38] (see also [24] for a modern treatment of the subject) found an integral representation of them and proved that they are exactly those functions which are operator-monotone, i.e. $f(A) \leq f(B)$, for any selfadjoint bounded operators $A \leq B$ on a Hilbert space. In particular, $f(t)=t^{s}(0 \leq s \leq 1)$ is operator-monotone. It turns out that this fact implies the following property that $G^{+}$shares, again, with Riemannian manifolds of non-positive curvature:

$$
d\left(\exp _{a}\left(s \exp _{a}^{-1}(x)\right), \exp _{a}\left(s \exp _{a}^{-1}(y)\right)\right) \leq s d(x, y)
$$

for all $s \in[0,1], x, y \in G^{+}$(see [19]).

In fact, the inequality can be written as

$$
\left\|\log \left(x^{-s / 2} y^{s} x^{-s / 2}\right)\right\| \leq s\left\|\log \left(x^{-1 / 2} y x^{-1 / 2}\right)\right\|,
$$

which can be proved using the inequality $\left\|x^{s} y^{s}\right\| \leq\|x y\|^{s}$, valid for all $s \in[0,1]$ and $x, y \in G^{+}$(see [30] for a proof that the last inequality is equivalent to Löwner's result that $0 \leq x \leq y$ implies $x^{s} \leq y^{s}$; the reader is also referred to [29] and [26] for more information on this type of inequalities).

We now show that Segal's inequality can be deduced from the inequality

$$
d\left(x^{s}, y^{s}\right) \leq s d(x, y), \quad s \in[0,1], x, y \in \mathcal{A}^{+}
$$

(which is $(2.3)$ for $a=1$ ).

Abbreviating $X=\log x, Y=\log y$ we get

$$
\begin{aligned}
x^{-s / 2} & =e^{-(s / 2) X}=1-(s / 2) X+o\left(s^{2}\right), \\
y^{s} & =e^{s Y}=1+s Y+o\left(s^{2}\right), \\
x^{-s / 2} y^{s} x^{-s / 2} & =1+s(Y-X)+o\left(s^{2}\right)
\end{aligned}
$$


and

Then

$$
\log \left(x^{-s / 2} y^{s} x^{-s / 2}\right)=s(Y-X)+o\left(s^{2}\right)
$$

$$
\lim _{s \rightarrow 0} \frac{d\left(x^{s}, y^{s}\right)}{s}=\lim _{s \rightarrow 0} \frac{\left\|\log \left(x^{-s / 2} y^{s} x^{-s / 2}\right)\right\|}{s}=\|X-Y\|
$$

so that $\|X-Y\| \leq d(x, y)$, which is an equivalent form of Segal's inequality.

Finally, we mention without proof another "non-positive curvature" property of $G^{+}$: given two geodesics $\gamma$ and $\delta$ on $G^{+}$, the function $t \mapsto d(\gamma(t), \delta(t))$ is convex [20] (see also [47] and [48]).

3. On the curvature tensor. Recall that, for $a \in G^{+}$and $X, Y, Z \in\left(T G^{+}\right)_{a}$, the curvature tensor is given by

$$
R(X, Y) Z=\frac{1}{4}\left(Z\left[a^{-1} X, a^{-1} Y\right]-\left[X a^{-1}, Y a^{-1}\right] Z\right) .
$$

We can easily determine the spectrum $\sigma(R(X, Y))$ in the algebra $\mathcal{L}(\mathcal{A})$. In fact, by a known result of Lumer and Rosenblum [39],

$$
\sigma(R(X, Y))=\sigma\left(\left[a^{-1} X, a^{-1} Y\right]\right)-\sigma\left(\left[X a^{-1}, Y a^{-1}\right]\right) .
$$

Now,

$$
a\left[a^{-1} X, a^{-1} Y\right] a^{-1}=\left[X a^{-1}, Y a^{-1}\right]
$$

so that

$$
\sigma(R(X, Y))=\sigma\left(\left[a^{-1} X, a^{-1} Y\right]\right)-\sigma\left(\left[a^{-1} X, a^{-1} Y\right]\right)
$$

Consider a faithful representation of $\mathcal{A}$ on the Hilbert space $\mathcal{H}$ and consider on $\mathcal{H}$ the scalar product $\langle,\rangle_{a}$ defined by

$$
\langle\xi, \eta\rangle_{a}=\langle a \xi, \eta\rangle \quad(\xi, \eta \in \mathcal{H}) .
$$

Then $a^{-1} X$ is $a$-selfadjoint (in the sense that $\left\langle a^{-1} X \xi, \eta\right\rangle_{a}=\left\langle\xi, a^{-1} X \eta\right\rangle_{a}$ ); analogously, $a^{-1} Y$ is $a$-selfadjoint.

As a consequence, $\left[a^{-1} X, a^{-1} Y\right]$ is $a$-skewsymmetric as an operator over $\mathcal{H}_{a}$. In particular,

$$
\sigma\left(\left[a^{-1} X, a^{-1} Y\right]\right) \subset i \mathbb{R} .
$$

(Observe that the spectrum depends neither on the representation nor on the scalar product on $\mathcal{H}$.) Finally, we get

$$
\sigma(R(X, Y)) \subset i \mathbb{R}
$$

Given $Y=Y^{*}$ the transformer $C_{Y}: X \mapsto 4 R(X, Y) Y$ can be written as

$$
C_{Y}=-\left(L_{Y a^{-1}}-R_{a^{-1} Y}\right)^{2},
$$

where $L_{Z}$ (resp. $R_{Z}$ ) denotes left (resp. right) multiplication by $Z$. Then $\sigma\left(C_{Y}\right)=$ $-\sigma\left(L_{Y a^{-1}}-R_{a^{-1} Y}\right)^{2}$. Now, $\sigma\left(L_{Y a^{-1}}\right)=\sigma\left(Y a^{-1}\right)=\sigma\left(a^{-1 / 2} Y a^{-1 / 2}\right) \subset \mathbb{R}($ see, for instance, [22]) and, analogously, $\sigma\left(R_{a^{-1} Y}\right) \subset \mathbb{R}$. Because $L_{Y a^{-1}}$ commutes with $R_{a^{-1} Y}$, we get

$$
\sigma\left(L_{Y a^{-1}}-R_{a^{-1} Y}\right) \subset \sigma\left(L_{Y a^{-1}}\right)-\sigma\left(R_{a^{-1} Y}\right) \subset \mathbb{R} .
$$


In general, we obtain:

Proposition. If $Y^{*}=Y$, then $\sigma\left(C_{Y}\right) \leq 0$. If $Y^{*}=-Y$, then $\sigma\left(C_{Y}\right) \geq 0$.

4. Operator means. An operator mean is a binary operation $m$ defined on $G^{+}$(or, more generally, on the closure of $G^{+}$) which satisfies

(i) $m(1,1)=1$,

(ii) $m(a, b) \leq m(c, d)$ if $a \leq c$ and $b \leq d$,

(iii) $c m(a, b) c \leq m(c a c, c b c)$,

(iv) if $a_{n} \downarrow a$ and $b_{n} \downarrow b$ then $m\left(a_{n}, b_{n}\right) \downarrow m(a, b)$.

The first non-trivial examples of these operations were, historically, the parallel sum $m(a, b)=2\left(a^{-1}+b^{-1}\right)^{-1}$ (see [1]) and the geometric mean $m(a, b)=$ $a^{1 / 2}\left(a^{-1 / 2} b a^{-1 / 2}\right)^{t} a^{1 / 2}$ (see [45]). By a result of Ando and Kubo [36], there is a bijective correspondence between operator means and operator-monotone functions $f: \mathbb{R}^{+} \rightarrow \mathbb{R}^{+}$, given by $m \mapsto f_{m}, f_{m}(t)=m(1, t)$ and $f \mapsto m_{f}$, $m_{f}(a, b)=a^{1 / 2} f\left(a^{-1 / 2} b a^{-1 / 2}\right) a^{1 / 2}$. Thus, the geodesic $\gamma$ of $G^{+}$joining $a$ to $b$ can be thought of as a parametrization between the left trivial mean $m_{l}(a, b)=a$ and the right trivial mean $m_{r}(a, b)=b$, the middle point corresponding to the geometrical mean of Pusz and Woronowicz. The velocity vector

$$
\dot{\gamma}(0)=a^{1 / 2} \log \left(a^{-1 / 2} b a^{-1 / 2}\right) a^{1 / 2}
$$

coincides with the so-called relative entropy $s(a, b)[28]$. Observe that $s(a, b)=$ $\exp _{a}^{-1}(b)$ (see Section 2). Segal's inequality shows that

$$
\|\log a-\log b\| \leq\left\|\log \left(a^{-1 / 2} b a^{-1 / 2}\right)\right\|=\left\|a^{-1 / 2} s(a, b) a^{-1 / 2}\right\| \leq\left\|a^{-1}\right\|\|s(a, b)\| .
$$

5. The geometry of the projections. Let $Q$ be the set of all idempotent elements of $\mathcal{A}$. $Q$ has a rich topological and geometrical structure which has been studied in [53], [25], [50], [41], [13], [14], [52] (see also [34] for a semigroup approach). We shall identify $Q$ with the set

$$
\left\{\varepsilon \in \mathcal{A}: \varepsilon^{2}=1\right\}
$$

by means of the affine transformation $x \mapsto 2 x-1$. With this identification in mind one can easily prove that $Q$ is an analytic submanifold of $\mathcal{A}$ and that

$$
(T Q)_{\varepsilon}=\{X \in \mathcal{A}: X \varepsilon+\varepsilon X=0\} .
$$

We can consider the polar decompositions of $\varepsilon \in Q: \varepsilon=\lambda^{2} \varrho, \varepsilon=\varrho \mu^{2}$, where $\lambda, \mu$ are positive and $\varrho$ is unitary (recall that the unitary part $\varrho$ is the same for both decompositions). It is easy to see that $\varrho \in P=\left\{x \in G: x^{*}=x=x^{-1}\right\}$ and that $\lambda \varrho=\varrho \lambda^{-1}$. Thus, as in the case of $G^{\mathrm{s}}$, we get a map $\pi: Q \rightarrow P$ whose fibers can be studied from a geometric viewpoint with rather complete results. In fact, define a Finsler structure on $Q$ as follows: if $\varepsilon \in Q$ and $X \in(T Q)_{\varepsilon}$, we decompose $\varepsilon=\lambda^{2} \varrho$ and define

$$
\|X\|_{\varepsilon}=\left\|\lambda X \lambda^{-1}\right\| .
$$


It turns out that the fibers $Q_{\varrho}=\pi^{-1}(\varrho)$ are geodesically complete: if $\varepsilon_{0}$ and $\varepsilon_{1}$ belong to $Q_{\varrho}$, they have polar decompositions of the form $\varepsilon_{0}=\lambda_{0} \varrho, \varepsilon_{1}=\lambda_{1} \varrho$ with $\lambda_{0}, \lambda_{1} \in G^{+}$, and $\lambda_{0} \varrho=\varrho \lambda_{0}^{-1}$ and $\lambda_{1} \varrho=\varrho \lambda_{1}^{-1}$; then the curve $\gamma(t)=$ $\lambda_{0}^{1 / 2}\left(\lambda_{0}^{-1 / 2} \lambda_{1} \lambda_{0}^{-1 / 2}\right)^{t} \lambda_{0} \varrho$ is a geodesic lying in $Q_{\varrho}$ and joining $\varepsilon_{0}$ and $\varepsilon_{1}$. For $\varepsilon, \varepsilon^{\prime} \in Q_{\varrho}$ the geodesic from $\varepsilon$ to $\varepsilon^{\prime}$ is the shortest curve in $Q$ joining them and it lies entirely in $Q_{\varrho}$ (see [14]; see also (5.4) for a remark leading to a simplification of the proof). As in the case of the selfadjoint invertible elements, the last result depends on a certain operator inequality. In fact, we need to prove that

$$
(T \pi)_{\varepsilon}:(T Q)_{\varepsilon} \rightarrow(T P)_{\varrho}
$$

decreases norms:

$$
\left\|(T \pi)_{\varepsilon} X\right\| \leq\|X\|_{\varepsilon}
$$

for all $\varepsilon \in Q, X \in(T Q)_{\varepsilon}$.

The original proof of (5.1) was quite complicated (see [15]) but A. Maestripieri [40] showed that (5.1) follows easily from (1.4). By a straightforward computation, inequality (5.1) can be transformed into

$$
\left\|S T S+S^{-1} T S^{-1}\right\| \geq 2\|T\|
$$

for every $T$ and a certain selfadjoint operator $S$. Using Pedersen's proof of (1.5), D. Stojanoff showed that (5.2) holds for every $T$ and every selfadjoint invertible $S$; moreover, he proved that (5.2) is equivalent to (1.4).

Proposition 5.3. Let $S$ and $T$ be bounded operators on a Hilbert space $\mathcal{H}$ and suppose that $S$ is invertible. Then

$$
\left\|S T S+S^{*-1} T S^{*-1}\right\| \geq 2\|T\| .
$$

Proof. Let

Then

$$
T_{1}=\left(\begin{array}{cc}
0 & T \\
T^{*} & 0
\end{array}\right) \quad \text { and } \quad S_{1}=\left(\begin{array}{cc}
S & 0 \\
0 & S^{-1}
\end{array}\right)
$$

$$
S_{1} T_{1} S_{1}^{-1}+S_{1}^{*-1} T_{1} S_{1}^{*-1}=\left(\begin{array}{cc}
0 & A \\
A^{*} & 0
\end{array}\right) \text {, where } A=S T S+S^{*-1} T S^{*-1} .
$$

Thus, $\left\|S_{1} T_{1} S_{1}^{-1}+S_{1}^{*-1} T_{1} S_{1}^{*}\right\|=\left\|S T S+S^{*-1} T S^{*-1}\right\|$; combining this with (1.5) we get the result.

Remark 5.4. As in the case of $G^{\text {s }}$, we can prove that

$$
d_{Q}\left(\varepsilon_{0}, \varepsilon_{1}\right)=d_{G^{+}}\left(\lambda_{0}, \lambda_{1}\right),
$$

where $\varepsilon_{0}=\lambda_{0} \varrho$ and $\varepsilon_{1}=\lambda_{1} \varrho$. The metric results on $G^{+}$produce, as before, analogous results on the fiber $Q_{\varrho}$; in particular, the geodesic

$$
\gamma(t)=\lambda_{0}^{1 / 2}\left(\lambda_{0}^{-1 / 2} \lambda_{1} \lambda_{0}^{-1 / 2}\right)^{t} \lambda_{0}^{1 / 2}
$$

is the shortest curve joining $\varepsilon_{0}$ and $\varepsilon_{1}$. 
6. Quadratic interpolation and geometry. (This section follows closely the paper [2].) Let $\mathcal{H}$ be a Hilbert space with scalar product $\langle$,$\rangle . For every$ positive invertible operator $a$ on $\mathcal{H}$ the scalar product $\langle,\rangle_{a}$ defined by

$$
\langle\xi, \eta\rangle_{a}=\langle a \xi, \eta\rangle \quad(\xi, \eta \in \mathcal{H})
$$

is equivalent to $\langle$,$\rangle ; moreover, by the Riesz representation theorem, every equiv-$ alent scalar product is one of these $\langle,\rangle_{a}$.

Together with the scalar product $\langle,\rangle_{a}$ we consider the norm \|\|$_{a}$ on $\mathcal{H}$ given by $\|\xi\|_{a}=\langle\xi, \xi\rangle_{a}^{1 / 2}(\xi \in \mathcal{H})$; the corresponding norm on $\mathcal{L}\left(\mathcal{N}_{a}\right)$ is given by

$$
\|X\|_{a}=\sup _{\|\xi\|_{a} \leq 1}\|X \xi\|_{a}=\left\|a^{1 / 2} X a^{-1 / 2}\right\| .
$$

(Observe that this norm is different from that defining the Finsler structure in Section 1, where $\|X\|_{a}$ is the norm of the sesquilinear form on $\mathcal{H}_{a}$ defined by $X$.)

We can consider the Banach-Mazur distance between two quadratic norms \|\|$_{a},\|\|_{b}$ on $\mathcal{H}$ :

$$
d\left(\|\|_{a},\|\|_{b}\right)=\max \left\{\log \sup _{\xi \neq 0} \frac{\|\xi\|_{a}}{\|\xi\|_{b}}, \log \sup _{\xi \neq 0} \frac{\|\xi\|_{b}}{\|\xi\|_{a}}\right\} .
$$

Surprisingly, this number is closely related to the geodesic distance $d(a, b)$.

Proposition 6.1. For every $a, b$ in $G^{+}$,

$$
2 d\left(\|\|_{a},\|\|_{b}\right)=d_{G^{+}}(a, b) .
$$

Proof. First, observe that

$$
\log \sup _{\xi \neq 0} \frac{\|\xi\|_{a}}{\|\xi\|_{b}}=\log \sup _{\xi \neq 0} \frac{\left\|a^{1 / 2} \xi\right\|}{\left\|b^{1 / 2} \xi\right\|}=\log \sup _{\xi \neq 0} \frac{\left\|a^{1 / 2} b^{-1 / 2} \xi\right\|}{\|\xi\|}=\log \left\|a^{1 / 2} b^{-1 / 2}\right\| ;
$$

analogously $\log \sup _{\xi \neq 0}\|\xi\|_{b} /\|\xi\|_{a}=\log \left\|b^{1 / 2} a^{-1 / 2}\right\|$.

On the other hand, $\|\log c\|=\max \left\{\log \|c\|, \log \left\|c^{-1}\right\|\right\}$ for all $c \in G^{+}$and $\left\|a^{-1 / 2} b a^{-1 / 2}\right\|=\left\|b^{1 / 2} a^{-1 / 2}\right\|^{2}$ for all $a, b \in G^{+}$. Thus,

$$
\begin{aligned}
d_{G^{+}}(a, b) & =\left\|\log \left(a^{-1 / 2} b a^{-1 / 2}\right)\right\|=\max \left\{\log \left\|a^{-1 / 2} b a^{-1 / 2}\right\|, \log \left\|a^{1 / 2} b^{-1} a^{1 / 2}\right\|\right\} \\
& =2 \max \left\{\log \left\|a^{-1 / 2} b^{1 / 2}\right\|, \log \left\|a^{1 / 2} b^{-1 / 2}\right\|\right\}=2 d\left(\|\|_{a},\|\|_{b}\right) .
\end{aligned}
$$

Given norms \|\|$_{a}$ and \|\|$_{b}$, every interpolation method provides a curve of norms \|\|$_{\gamma(t)}(0 \leq t \leq 1)$ with some continuity properties and such that $\gamma(0)=a$ and $\gamma(1)=b$. It turns out that the so-called real and complex methods both produce the same curve of norms \|\|$_{\gamma(t)}$, where $\gamma$ is exactly the geodesic from $a$ to $b, \gamma(t)=a^{1 / 2}\left(a^{-1 / 2} b a^{-1 / 2}\right)^{t} a^{1 / 2}$. There is a nice proof by Semmes ([47], [48], [12]) of the convexity of the function $f(t)=d\left(\|\|_{\gamma(t)},\|\|_{\delta(t)}\right)$, which is essentially different from that of [20]. Unfortunately, Semmes' methods are only valid for finite-dimensional spaces: using the notion of superharmonicity and subharmonicity of norm-valued functions $z \mapsto\|\|_{z}$, he shows that the distance $d\left(||_{z},\|\|_{z}\right)$ is subharmonic, which means, when $z$ is restricted to the real line, that $d\left(||_{z},\|\|_{z}\right)$ is convex. 
Re m a r k. The geodesics on the fibers $G_{\varrho}^{\mathrm{s}}$ can be thought of as an interpolation method of Krein structures (see [2] for details).

7. Other spaces. Although the main results described in this paper are concerned with the spaces $G^{\mathrm{s}}$ and $Q$, there are several other spaces having a geometrical structure which have been extensively studied. Historically, the first systematic account of Banach-Lie groups of operators has been given by Pierre de la Harpe (see [33], which also contains a complete bibliography up to 1972). Later on, the papers of Atkin developed a study of Finsler structures on classical groups [9], [10].

In [13] there is a geometric study of the space $Q_{n}$ of $n$-tuples $\left(q_{1}, \ldots, q_{n}\right)$ of idempotents of $\mathcal{A}$ such that $q_{i} q_{j}=\delta_{i j} q_{i}$ and $\sum_{i=0}^{n} q_{i}=1$. This space can be identified with the set of solutions of the equation $p(X)=0$, where $p$ is a complex polynomial of degree $n$ with only simple zeros. The space $Q_{n}$ can be seen in a number of ways. For instance, Martin [42] considers $Q_{n}$ as the space of representations of $\mathbb{Z}_{n}$ in $\mathcal{A}$; he studies the geometry of the space of representations of a compact group on a $C^{*}$-algebra $\mathcal{A}$; on the other hand, Andruchow, Recht and Stojanoff [5] study the space of spectral measures of a Boolean algebra on $\mathcal{A}$. Under their viewpoint, $Q_{n}$ can be seen as the set of spectral measures of the Boolean algebra generated by $n$ atoms with values in $\mathcal{A}$. Other papers by Andruchow and Stojanoff (see [6]-[8]) study the geometry of orbits of operators under the action of the whole group $G$ or under the action of the unitary group $\mathcal{U}$ (see also Lorentz' paper [37]). The study of the geometry of the space of relatively regular operators has been initiated by B. Gramsch [31] and continued in [21]. Finally, we mention the work of A. Maestripieri [40], who extended the results of [14] and [16] in the following sense: instead of considering the spaces $\left\{\varepsilon: \varepsilon^{2}=1\right\}$ and $\left\{a \in G: a^{*}=a\right\}$ she studies the spaces $\left\{\varepsilon: \varepsilon^{2}=\sigma\right\}$ and $\left\{a \in G: a^{*} a^{-1}=\sigma\right\}$, where $\sigma$ is an arbitrary element of $G$. The results in this context are similar to those obtained in [14] and [16] for the case $\sigma=1$. The study of metric results for the spaces mentioned in this section remains an open problem: apparently, the main point seems to be to find the right Finsler structure.

\section{References}

[1] W. N. Anderson and G. E. Trapp, Shorted operators II, SIAM J. Appl. Math. 28 (1975), 60-71.

[2] E. Andruchow, G. Corach, M. Milman and D. Stojanoff, Geodesics and interpolation, preprint.

[3] E. Andruchow, G. Corach and D. Stojanoff, A geometric characterization of nuclearity and injectivity, preprint.

[4] E. Andruchow, L. A. Fialkow, D. A. Herrero, M. B. Pecuch and D. Stojanoff, Joint similarity orbits with local cross sections, Integral Equations Operator Theory 13 (1990), 1-48. 
[5] E. Andruchow, L. Recht and D. Stojanoff, The space of spectral measures in a homogeneous reductive space, ibid. 16 (1993), 1-14.

[6] E. Andruchow and D. Stojanoff, Nilpotent operators and systems of projections, J. Operator Theory 20 (1988), 359-374.

[7] - , - Differentiable structure of similarity orbits, ibid. 21 (1989), 349-366.

[8] -, -, Geometry of unitary orbits, ibid. 25 (1991), 25-41.

[9] C. J. Atkin, The Finsler geometry of groups of isometries of Hilbert space, J. Austral. Math. Soc. Ser. A 42 (1987), 196-222.

[10] - The Finsler geometry of certain covering groups of operator groups, Hokkaido Math. J. 18 (1989), 45-77.

[11] J. Bergh and J. Löfström, Interpolation Spaces. An Introduction, Springer, New York, 1976.

[12] R. Coifman and S. Semmes, Interpolation of Banach spaces, Perron processes and Yang-Mills, Amer. J. Math. 115 (1993), 243-278.

[13] G. Corach, H. Porta and L. Recht, Differential geometry of systems of projections in Banach algebras, Pacific J. Math. 140 (1990), 209-228.

[14] - , - - , The geometry of the spaces of projections in $C^{*}$-algebras, Adv. in Math. 101 (1993), 59-77.

[15] - , - - - Two $C^{*}$-algebra inequalities, in: Analysis in Urbana, Proc. Special Year in Modern Analysis at the University of Illinois, E. Berkson and J. Uhl (eds.), Cambridge University Press, 1989, 141-143.

[16] - - - - - The geometry of spaces of selfadjoint invertible elements of a $C^{*}$-algebra, Integral Equations Operator Theory 16 (1993), 333-359.

[17] —, - - - An operator inequality, Linear Algebra Appl. 142 (1990), 153-158.

[18] - , - - , A geometric interpretation of the inequality $\left\|e^{X+Y}\right\| \leq\left\|e^{X / 2} e^{Y} e^{X / 2}\right\|$, Proc. Amer. Math. Soc. 115 (1992), 229-231.

[19] - - - - , Geodesics and operator means in the space of positive operators, Internat. J. Math. 4 (1993), 193-202.

[20] - , - - Convexity of the geodesic distance on spaces of positive operators, Illinois J. Math., to appear.

[21] - , - - Differential geometry of spaces of relatively regular operators, Integral Equations Operator Theory 13 (1990), 771-794.

[22] R. Curto, Applications of several complex variables to multiparameter spectral theory, in: Surveys of Some Recent Results in Operator Theory, Vol. II, J. B. Conway and B. B. Morrel (eds.), Longman, London, 1988, 25-90.

[23] Ch. Davis, The norm of the Schur product operation, Numer. Math. 4 (1962), 343-344.

[24] W. F. Donoghue, Monotone Matrix Functions and Analytic Continuation, Springer, Berlin, 1974.

[25] J. Esterle, Polynomial connections between projections in Banach algebras, Linear Algebra Appl. 15 (1983), 253-254.

[26] J. Fujii, M. Fujii, T. Furuta and R. Nakamoto, Norm inequalities equivalent to Heinz inequality, Proc. Amer. Math. Soc. 118 (1993), 827-830.

[27] —, - - - - , Norm inequalities related to McIntosh type inequality, Nihonkai J. Math. 3 (1992), 67-72.

[28] J. I. Fujii and E. Kamei, Relative operator entropy in noncommutative information theory, Math. Japon. 34 (1989), 341-348.

[29] M. Fujii and R. Nakamoto, On certain norm inequalities, ibid. 38 (1993), 79-81.

[30] T. Furuta, Norm inequalities equivalent to Löwner-Heinz theorem, Rev. Math. Phys. 1 (1989), 135-137.

[31] B. Gramsch, Relative Inversion in der Störungstheorie von Operatoren und $\psi$-Algebren, Math. Ann. 269 (1984), 27-71. 
[32] U. Haagerup, Solution of the similarity problem for cyclic representations of $C^{*}$-algebras, Ann. of Math. 118 (1983), 215-240.

[33] P. de la Harpe, Classical Banach-Lie Algebras and Banach-Lie Groups of Operators in Hilbert Space, Lecture Notes in Math. 285, Springer, Berlin, 1972.

[34] J. P. Holmes, The structure of the set of idempotents in a Banach algebra, Illinois J. Math. 36 (1992), 102-115.

[35] M. Kobayashi and K. Nomizu, Foundations of Differential Geometry, Interscience, New York, 1969.

[36] F. Kubo and T. Ando, Means of positive linear operators, Math. Ann. 246 (1980), 205224.

[37] K. Lorentz, On the local structure of the similarity orbits of Jordan elements in operator algebras, Ann. Univ. Sarav. Ser. Math. 2 (3) (1989).

[38] K. Löwner, Über monotone Matrixfunktionen, Math. Z. 38 (1934), 177-216.

[39] G. Lumer and M. Rosenblum, Linear operator equations, Proc. Amer. Math. Soc. 10 (1959), 32-41.

[40] A. Maestripieri, On the geometry of the set of square roots of elements in $C^{*}$-algebras, Integral Equations Operator Theory, to appear.

[41] E. Makai, Jr. and J. Zemánek, On polynomial connections between projections, Linear Algebra Appl. 126 (1985), 91-94.

[42] M. Martin, Projective representations of compact groups in $C^{*}$-algebras, in: Oper. Theory: Adv. Appl. 43, Birkhäuser, 1990, 237-253.

[43] G. Pólya und G. Szegö, Aufgaben und Lehrsätze aus der Analysis, Springer, Berlin, 1925.

[44] H. Porta and L. Recht, Minimality of geodesics in Grassmann manifolds, Proc. Amer. Math. Soc. 100 (1987), 464-466.

[45] W. Pusz and S. L. Woronowicz, Functional calculus for sesquilinear forms and the purification map, Rep. Math. Phys. 8 (1975), 159-170.

[46] I. Segal, Notes toward the construction of nonlinear relativistic quantum fields III, Bull. Amer. Math. Soc. 75 (1969), 1390-1395.

[47] S. Semmes, Interpolation of Banach spaces, differential geometry and differential equations, Rev. Mat. Iberoamericana 4 (1988), 155-176.

[48] -, Complex Monge-Ampère and symplectic manifolds, preprint.

[49] N. Steenrod, The Topology of Fiber Bundles, Princeton University Press, Princeton, 1951.

[50] M. Tremon, Polynômes de degré minimum connectant deux projections dans une algèbre de Banach, Linear Algebra Appl. 64 (1985), 115-132.

[51] M. Walter, On the norm of the Schur product, ibid. 79 (1986), 209-213.

[52] D. R. Wilkins, The Grassmann manifold of a $C^{*}$-algebra, Proc. Roy. Irish Acad. 90A (1990), 99-116.

[53] J. Zemánek, Idempotents in Banach algebras, Bull. London Math. Soc. 11 (1979), $177-$ 183. 